\title{
ON AUTOMORPHISMS OF MATRIX INVARIANTS
}

\author{
ZINOVY REICHSTEIN
}

\begin{abstract}
Let $Q_{m, n}$ be the space of $m$-tuples of $n \times n$-matrices modulo the simultaneous conjugation action of $P G L_{n}$. Let $Q_{m, n}(\tau)$ be the set of points of $Q_{m, n}$ of representation type $\tau$. We show that for $m \geq n+1$ the group Aut $\left(Q_{m, n}\right)$ of representation type preserving algebraic automorphisms of $Q_{m, n}$ acts transitively on each $Q_{m, n}(\tau)$. Moreover, the action of $\operatorname{Aut}\left(Q_{m, n}\right)$ on the Zariski open subset $Q_{m, n}(1, n)$ of $Q_{m, n}$ is $s$-transitive for every positive integer $s$. We also prove slightly weaker analogues of these results for all $m \geq 3$.
\end{abstract}

\section{INTRODUCTION}

Let $M_{n}(k)^{m}$ be the set of $m$-tuples of $n \times n$-matrices over an algebraically closed field $k$ of characteristic 0 . The group $P G L_{n}(k)$ acts on $M_{n}(k)^{m}$ by conjugation. Let $Q_{m, n}$ be the algebraic quotient for this action.

A point $x$ of $Q_{m, n}$ can be lifted to an $m$-tuple of matrices $\left(X_{1}, \ldots, X_{m}\right) \in$ $M_{n}(k)$ such that the representation

$$
\phi_{s s}(x): k\left\{u_{1}, \ldots, u_{m}\right\} \rightarrow M_{k}(k), \quad u_{i} \rightarrow X_{i},
$$

is semisimple. Moreover, this representation is unique up to equivalence. We say that $x$ has representation type $\tau=\left(e_{1}, d_{1} ; \ldots ; e_{r}, d_{r}\right)$ if $\phi(x)$ is the sum of $r$ irreducible representations $\phi_{i}$ of dimension $d_{i}$ and multiplicity $e_{i}$. The set of all points of $Q_{m, n}$ of representation type $\tau$ will be denoted by $Q_{m, n}(\tau)$. We remark that $Q_{m, n}(1, n)$ is Zariski open and dense in $Q_{m, n}$. For further details, see $\S 2$.

Note that $Q_{m, 1}$ is trivially isomorphic to the affine space $k^{m}$. In general, however, $Q_{m, n}$ is a very complicated affine variety, both locally and globally.

The local étale structure of $Q_{m, n}$ was studied by Le Bruyn and Procesi [9]. They showed that if $m, n \geq 2$ and $(m, n) \neq(2,2)$ then $Q_{m, n}(1, n)$ is precisely the smooth locus of $Q_{m, n}$. They also gave a description of the étale neighborhood of a point of $Q$ in terms of representations of quivers; see [9, $\S \S 0$ and II.1]. The following observation is an immediate consequence of this description.

1.1 Proposition. Étale neighborhoods of points of the same representation type are isomorphic.

Received by the editors June 3, 1991 and, in revised form, September 10, 1991.

1991 Mathematics Subject Classification. Primary 16R30, 14L30, 14E09; Secondary 16 S10.

Key words and phrases. Matrix invariants, representation type, algebraic automorphisms. 
Since $Q_{m, n}$ is defined as the quotient of an affine space, it is unirational. Procesi and Formanek showed that $Q_{m, n}$ is rational for $n=2,3,4$; see [11, 4, and 5]. Bessenrodt and LeBruyn [2] proved that $Q_{m, 5}$ and $Q_{m, 7}$ are stably rational. Katsylo [7] and Schofield [15] showed that $Q_{m, a b}$ is stably birational to $Q_{m, a} \times Q_{m, b}$ if $a$ and $b$ are relatively prime. This, in particular, implies that $Q_{m, n}$ is stably rational whenever $n$ divides 420 . The rationality or stable rationality of $Q_{m, n}$ for other values of $n$ is an open problem. For a survey of this problem and related questions we refer the reader to [6 and 8].

The purpose of this paper is to demonstrate that for $m \geq 3 \quad Q_{m, n}$ has a very large automorphism group. Let $\operatorname{Aut}\left(Q_{m, n}\right)$ be the group of algebraic automorphisms of $Q_{m, n}$ which preserve the representation type. Our main results are as follows.

1.2 Theorem. Let $\tau=\left(e_{1}, d_{1} ; \ldots ; e_{r}, d_{r}\right)$. If $m \geq \max \left\{d_{1}, \ldots, d_{r}\right\}+1$ then $\operatorname{Aut}\left(Q_{m, n}\right)$ acts transitively on $Q_{m, n}(\tau)$. In particular, if $m \geq n+1$ then $\operatorname{Aut}\left(Q_{m, n}\right)$ acts transitively on every $Q_{m, n}(\tau)$.

1.3 Theorem. Let $m \geq n+1$. Then $\operatorname{Aut}\left(Q_{m, n}\right)$ acts s-transitively on $Q_{m, n}(1, n)$ for every integer $s \geq 1$.

Note that if $n=1$ then $Q_{m, n}=Q_{m, n}(1, n)=k^{m}$, and Theorem 1.3 says that the group of algebraic automorphisms of $k^{m}$ acts on $k^{m} s$-transitively for any $s \geq 1$; see Tineorem 3.1 .

We prove slightly weaker analogues of Theorems 1.2 and 1.3 for all $m \geq 3$.

1.4 Theorem. Let $m \geq 3$. For every representation type $\tau$ there is an open $\operatorname{Aut}\left(Q_{m, n}\right)$-invariant subset $U_{m, n}(\tau)$ of $Q_{m, n}(\tau)$ on which $\operatorname{Aut}\left(Q_{m, n}\right)$ acts stransitively.

1.5 Theorem. There exists a nonempty Zariski-open subset $U_{m, n}$ of $Q_{m, n}$ on which $\operatorname{Aut}\left(Q_{m, n}\right)$ acts s-transitively for every integer $s \geq 1$.

Theorems 1.2 and 1.4 can be thought of as generalizations of Proposition 1.1. On the other hand, Theorems 1.3 and 1.5 may be viewed as properties of the global geometry of $Q_{m, n}$.

The rest of the paper is structured as follows. In $\S 2$ we introduce some notions and results which are used in subsequent sections. In $\S 3$ we prove the main theorems for $n=1$. In $\S \S 4$ and 5 we discuss a way of constructing elements of $\operatorname{Aut}\left(Q_{m, n}\right)$ and outline a proof of Theorems 1.2-1.5. The arguments we present there rely on Theorems 4.3 and 4.4 about polynomial equivalence of representations of the free algebra. These results are proved in $\S \S 6,7$, and 8 .

\section{Preliminaries}

2.1 Affine quotients. Let $G$ be a reductive group. Given a finite-dimensional representation $G \rightarrow \mathrm{GL}_{N}(k)$ and a $G$-invariant affine variety $X \subset k^{N}$, we define the quotient variety $X / G$ as the spectrum of the ring $k[X]^{G}$ of $G$ invariant regular functions on $X$. The quotient map $\pi: X \rightarrow X / G$ is then induced by the inclusion of rings $k[X]^{G} \hookrightarrow k[X]$. By a theorem of Hilbert $X / G$ is an affine variety. The quotient map $\pi$ is the categorical quotient for the $G$-action on $X$. That is, any $G$-equivariant map $f: X \rightarrow T$ into an affine variety $T$ with the trivial $G$-action factors through a unique morphism $X / G \rightarrow T$; see [10, Theorem 1.1]. Hence, we have the following 
2.2 Lemma. Let $F: X \rightarrow Y$ be a G-equivariant morphism of affine varieties. Then $F$ descends to a map of the quotient varieties, i.e. there exists a map $f: X / G \rightarrow Y / G$ such that the diagram

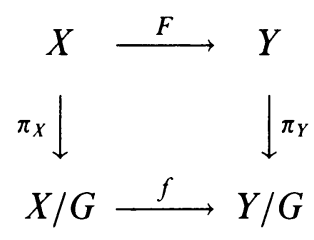

commutes. by

We shall be primarily interested in the action of $P G L_{n}(k)$ on $M_{k}(k)^{m}$ given

$$
\begin{gathered}
P G L_{n}(k) \times M_{n}(k)^{m} \rightarrow M_{n}(k)^{m}, \\
g\left(X_{1}, \ldots, X_{m}\right) \rightarrow\left(g^{-1} X_{1} g, \ldots, g^{-1} X_{m} g\right) .
\end{gathered}
$$

The coordinate ring of $M_{n}(k)^{m}$ is the polynomial ring $k\left[x_{i j}^{l}\right]$ where $x_{i j}^{l}$ is the $(i, j)$-entry of the $l$ th matrix $X_{l}$. We shall denote the quotient map for this action by $\pi_{m, n}:\left(M_{n}(k)\right)^{m} \rightarrow Q_{m, n}$. The ring of invariants $C_{m, n}=k\left[x_{i j}^{(l)}\right]^{P G L_{n}}$ is generated by elements of the form $\operatorname{tr}\left(X_{i_{1}} \cdots X_{i_{N}}\right)$; see [17, Theorem 1], [12, Theorem 1.3].

2.3 Representations. Let $M$ be a $k$-algebra. By a representation (resp. an irreducible representation) of $k\left\{u_{1}, \ldots, u_{m}\right\}$ in $M$ we mean a $k$-algebra homomorphism (resp. a surjective $k$-algebra homomorphism)

$$
\rho: k\left\{u_{1}, \ldots, u_{m}\right\} \rightarrow M .
$$

Such a representation can be viewed as an $m$-tuple of elements (resp. generators) of $M$. If $\rho\left(u_{i}\right)=X_{i}$ we will write $\rho=\left(X_{1}, \ldots, X_{m}\right)$.

Two representations $\rho_{1}: k\left\{u_{1}, \ldots, u_{m}\right\} \rightarrow M_{1}$ and $\rho_{2}: k\left\{u_{1}, \ldots, u_{m}\right\} \rightarrow$ $M_{2}$ are said to be equivalent if there exists an isomorphism $f: M_{1} \rightarrow M_{2}$ such that $\rho_{2}=f \circ \rho_{1}$. We write $\rho_{1} \approx \rho_{2}$. The direct sum $\rho_{1} \oplus \rho_{2}$ is a representation of $k\left\{u_{1}, \ldots, u_{m}\right\}$ in $M_{1} \times M_{2}$ defined in the obvious way. Up to equivalence, the operation $\oplus$ is commutative and associative. A representation is called semisimple if its image is a semisimple $k$-algebra, i.e. a product of the matrix algebras over $k$.

2.4 Lemma. For $i=1, \ldots, r$ let $\phi_{i}$ be a representation of $k\left\{u_{1}, \ldots, u_{m}\right\}$ in the matrix algebra $M_{d_{i}}(k)$. Then $\rho=\rho_{1} \oplus \cdots \oplus \rho_{r}$ is irreducible if and only if $\rho_{1}, \ldots, \rho_{r}$ are irreducible and pairwise inequivalent.

Proof. The lemma is a consequence of [1, Theorem 9.2] and the Chinese Remainder Theorem [14, Lemma 1.7.15]. Q.E.D.

2.5 The representation type. Let $\phi=\left(X_{1}, \ldots, X_{m}\right)$ be a representation of $k\left\{u_{1}, \ldots, u_{m}\right\}$ in the matrix algebra $M_{n}(k)$. Recall that a strictly increasing sequence of vector subspaces of $k^{n}$,

$$
(0)=V_{0} \subset V_{1} \subset \cdots \subset V_{q}=k^{n}
$$

is called a composition series for $\phi$ if each $V_{i}$ is invariant for $X_{1}, \ldots, X_{m}$ and the induced representations $\phi_{i}: k\left\{u_{1}, \ldots, u_{m}\right\} \rightarrow \operatorname{End}\left(V_{i} / V_{i-1}\right)$ are irreducible 
for $i=1, \ldots, q$. The (unordered) sequence of equivalence classes of representations $\phi_{1}, \ldots, \phi_{q}$ of $k\left\{u_{1}, \ldots, u_{m}\right\}$ in matrix algebras is independent of the choice of the composition series. Artin [1] showed that it depends only on $\pi_{m, n}\left(X_{1}, \ldots, X_{m}\right)$ and that, in fact, such sequences (with the dimensions summing up to $n$ ) are in bijective correspondence with points of $Q_{m, n}$. In other words, if in some basis of $k^{n}$,

$$
\phi=\left(\begin{array}{lll}
\phi_{1} & & * \\
& \ddots & \\
0 & & \phi_{r}
\end{array}\right)
$$

with all $\phi_{i}$ irreducible then the unordered sequence $\phi_{1}, \ldots, \phi_{q}$ depends only on $x=\pi_{m, n}\left(X_{1}, \ldots, X_{m}\right) \in Q_{m, n}$.

The semisimple representation associated to $\phi($ or $x)$ is then defined by

$$
\phi_{s s}=\phi_{s s}(x)=\left(\begin{array}{ccc}
\phi_{1} & & 0 \\
& \ddots & \\
0 & & \phi_{r}
\end{array}\right) \text {. }
$$

Let $\phi_{i_{1}}, \ldots, \phi_{i_{r}}$ be a maximal set of pairwise inequivalent representations among the $\phi_{i}$. If $\phi_{i_{j}}$ is of dimension $d_{j}$ and it occurs $e_{j}$ times then we define the representation type of $x$ (or $\phi$ ) to be the unordered $r$-tuple of pairs $\left(e_{j}, d_{j}\right)$. We write

$$
\tau(x)=\tau(\phi)=\tau\left(X_{1}, \ldots, X_{m}\right)=\left(e_{1}, d_{1} ; \cdots ; e_{r}, d_{r}\right) .
$$

The associated reduced semisimple representation of $x$ is given by

$$
\varphi(x)=\phi_{i_{1}} \oplus \cdots \oplus \phi_{i_{r}}: k\left\{u_{1}, \ldots, u_{m}\right\} \rightarrow M_{d_{1}} \times \cdots \times M_{d_{r}} .
$$

It is well defined up to equivalence. By Lemma $2.4 \varphi(x)$ is always irreducible.

An unordered $r$-tuple of pairs of positive integers $\tau=\left(e_{1}, d_{1} ; \ldots ; e_{r}, d_{r}\right)$ is said to be a representation type (for $n \times n$-matrices) if $e_{1} d_{1}+\cdots+e_{r} d_{r}=n$. There is a partial order on the set of representation types. We can obtain a smaller representation type by subdividing a diagonal block in (3) into two or by declaring two previously inequivalent blocks equivalent. In particular, for any representation type $\tau$ we have $(n, 1) \leq \tau \leq(1, n)$. Another equivalent definition, purely in terms of the integers $e_{i}$ and $d_{i}$, is given in $[9, \S I I .1]$. See also $[13, \S 7]$ for a characterization of the representation type in terms of algebras with trace.

The following lemma can be easily derived from either definition.

\subsection{Lemma. Let}

$$
\rho_{1}: k\left\{u_{1}, \ldots, u_{a}\right\} \rightarrow M_{n}(k) \text { and } \rho_{2}: k\left\{u_{1}, \ldots, u_{b}\right\} \rightarrow M_{n}(k)
$$

be representations such that $\rho_{1}\left(k\left\{u_{1}, \ldots, u_{a}\right\}\right) \subset \rho_{2}\left(k\left\{u_{1}, \ldots, u_{b}\right\}\right)$. Then $\tau\left(\rho_{1}\right) \leq \tau\left(\rho_{2}\right)$.

We define $Q_{m, n}(\tau)$ to be the set of points $Q_{m, n}$ of representation type $\tau$. The following fact is a consequence of a theorem of Schwarz [16, Lemma 5.5] for affine group actions. 
2.7 Theorem (Le Bruyn, Procesi [9, Theorem II.1.1]). The Zariski closure of $Q_{m, n}(\tau)$ is the union of $Q_{m, n}(\nu)$ taken over all $\nu \leq \tau . Q_{m, n}(\tau)$ is irreducible and open in its closure.

2.8 Corollary. The set of $m$-tuples $\left(X_{1}, \ldots, X_{m}\right)$ of $n \times n$-matrices of representation type $\geq \tau$ is open in $M_{n}(k)^{m}$.

Proof. Denote the set of points of $Q_{m, n}$ of representation type $\geq \tau$ (resp. $\leq \tau$ ) by $Q_{m, n}(\geq \tau)$ (resp. $Q_{m, n}(\leq \tau)$ ). By Theorem $2.7 Q_{m, n}(\leq \nu)$ is closed for every representation type $\nu$. Since there are only finitely many representation types, the set

$$
Q_{m, n}(\geq \tau)=Q_{m, n} \backslash \bigcup_{\nu \geq \tau} Q_{m, n}(\leq \nu)
$$

is open as the complement of a finite union of closed sets. The set we are interested in is the preimage of $Q_{m, n}(\geq \tau)$ under $\pi_{m, n}$. Q.E.D.

\subsection{Saturated representations.}

2.10 Lemma. A finite-dimensional semisimple algebra $M$ is generated by two elements.

Proof. By Lemma 2.4 it is enough to show that there are infinitely many pairwise inequivalent irreducible representations $\rho: k\left\{u_{1}, u_{2}\right\} \rightarrow M_{n}(k)$. This follows from Lemma 6.2(a). Q.E.D.

A representation $\rho: k\left\{u_{1}, \ldots, u_{m}\right\} \rightarrow M$ is called saturated if $\rho\left(u_{1}\right), \ldots$, $\rho\left(u_{m-1}\right)$ generate $M$ as a $k$-algebra.

2.11 Lemma. For any representation type $\tau$ the set of points $x$ whose reduced semisimple representation $\varphi(x)$ is saturated, is open in $Q_{m, n}(\tau)$. For $m \geq 3$ this set is nonempty.

Proof. The nonemptiness assertion is a consequence of Lemma 2.10. Let $F$ : $M_{n}(k)^{m} \rightarrow M_{n}(k)^{m-1}$ be the $P G L_{n}$-equivariant map given by $\left(X_{1}, \ldots, X_{m}\right) \rightarrow$ $\left(X_{1}, \ldots, X_{m-1}\right)$. By Lemma 2.2 this map descends to $f: Q_{m, n} \rightarrow Q_{m-1, n}$. Taking $\rho_{1}=\left(X_{1}, \ldots, X_{m-1}\right)$ and $\rho_{2}=\left(X_{1}, \ldots, X_{m}\right)$ in Lemma 2.6 we see that $\tau(f(x)) \leq \tau(x)$ for any $x \in Q_{m, n}$. Hence,

$$
f Q_{m, n}(\tau) \subset \bigcup_{\nu \leq \tau} Q_{m-1, n}(\nu),
$$

and the set we are interested in is $f^{-1} Q_{m-1, n}(\tau) \cap Q_{m, n}(\tau)$. The lemma now follows from Theorem 2.7. Q.E.D.

\section{AUTOMORPHISMS OF $k^{m}$}

In this section we shall prove Theorems $1.2-1.5$ in the case $n=1$. In this case every point of $Q_{m, 1}=M_{1}(k)^{m}=k^{m}$ is of representation type $(1,1)$, and Theorems $1.2-1.5$ reduce to the following.

3.1 Theorem. For $m \geq 2$ the group $\operatorname{Aut}\left(k^{m}\right)$ of polynomial automorphisms of the affine space $k^{m}$ acts on $k^{m}$ s-transitively for positive integer $s$.

Note that every automorphism of $k^{1}$ is affine, i.e. of the form $x \rightarrow a x+b$. Hence, $\operatorname{Aut}\left(k^{1}\right)$ is 2-transitive but not 3-transitive. We also remark that our proof will work for any infinite base field $k$. 
Proof. Fix $s$ distinct elements $c_{1}, \ldots, c_{s}$ of the base field $k$. It is enough to show that for any $s$-tuple of distinct points $x_{1}, \ldots, x_{s} \in k^{m}$ there exists a $g \in \operatorname{Aut}\left(k^{m}\right)$ and a nonzero $e \in k^{m}$ so that $g\left(x_{i}\right)=c_{i} e$. Indeed, if $g\left(x_{i}\right)=c_{i} e$ and $h\left(y_{i}\right)=c_{i} f$ for some $0 \neq e, f \in k^{m}$ and $g, h \in \operatorname{Aut}\left(k^{m}\right)$ then $y_{i}=$ $h^{-1} \circ L \circ g\left(x_{i}\right)$ where $L$ is any linear transformation of $k^{m}$ taking $e$ to $f$.

Choose a basis $u_{1}, \ldots, u_{m}$ for the space of linear forms on $k^{m}$ so that $u_{1}\left(x_{i}\right) \neq u_{1}\left(x_{j}\right)$ for any $1 \leq i<j \leq s$. In this coordinate system $x_{i}=$ $\left(x_{1 i}, \ldots, x_{m i}\right)$ where $x_{11}, \ldots, x_{1 s}$ are distinct. Thus for any $a_{1}, \ldots, a_{s} \in$ $k$ there exists a polynomial $f(t)$ in one variable such that $f\left(x_{1 i}\right)=a_{i}$ for $i=1, \ldots, s$. In particular, there exist polynomials $f_{2}(t), \ldots, f_{m}(t)$ such that $f_{2}\left(x_{1 i}\right)=c_{i}-x_{2 i}$ and $f_{j}\left(x_{1 i}\right)=-x_{j i}$ for $i=1, \ldots, s, j=3, \ldots, m$. Let $h_{1}$ be the automorphism of $k^{m}$ given by

$$
\begin{aligned}
& u_{1} \rightarrow u_{1}, \\
& u_{2} \rightarrow u_{2}+f_{2}\left(u_{1}\right), \\
& \vdots \\
& u_{m} \rightarrow u_{m}+f_{m}\left(u_{1}\right) .
\end{aligned}
$$

Then $h_{1}\left(x_{i}\right)=\left(x_{11}, c_{i}, 0,0, \ldots, 0\right)$ for $i=1, \ldots, s$. Now let $h_{2}$ be the automorphism of $k^{m}$ given by

$$
\begin{aligned}
& u_{1} \rightarrow u_{1}-f\left(u_{2}\right), \\
& u_{2} \rightarrow u_{2}, \\
& \vdots \\
& u_{m} \rightarrow u_{m},
\end{aligned}
$$

where $f\left(c_{i}\right)=x_{1 i}$ for $i=1, \ldots, n$. Then for $i=1, \ldots, s, h_{2} \circ h_{1}\left(x_{i}\right)=$ $\left(0, c_{i}, 0, \ldots, 0\right)$, as desired. Q.E.D.

Theorem 3.1 has the following algebraic formulation. Let $M=k \times \cdots \times k$ ( $s$ times) and let $\rho_{1}$ and $\rho_{2}: k\left[x_{1}, \ldots, x_{m}\right] \rightarrow M$ be surjective $k$-algebra homomorphisms. Then there exists an automorphism of the polynomial algebra $\sigma: k\left[x_{1}, \ldots, x_{m}\right] \rightarrow k\left[x_{1}, \ldots, x_{m}\right]$ such that $\rho_{2}=\rho_{1} \circ \sigma$.

Observe that in the above statement we can replace the commutative polynomial algebra $k\left[x_{1}, \ldots, x_{m}\right]$ by the noncommutative polynomial algebra $k\left\{u_{1}, \ldots, u_{m}\right\}$; our proof will work without any changes. We record these observations below.

3.2 Definition. Let $M$ be a $k$-algebra and let $\rho_{1}$ and $\rho_{2}: k\left\{u_{1}, \ldots, u_{m}\right\} \rightarrow$ $M$ be representations. We say that $\rho_{1}$ and $\rho_{2}$ are polynomially equivalent if there exists an automorphism $\sigma: k\left\{u_{1}, \ldots, u_{m}\right\} \rightarrow k\left\{u_{1}, \ldots, u_{m}\right\}$ such that $\rho_{2}=\rho_{1} \circ \sigma$.

Since a representation of $k\left\{u_{1}, \ldots, u_{m}\right\}$ in $M$ uniquely determines an $m$ tuple of elements in $M$ and vice versa, we shall also speak of polynomial equivalence $m$-tuples of elements of $M$.

3.3 Proposition. If $s \geq 1$ and $m \geq 2$ then any two irreducible representations of $k\left\{u_{1}, \ldots, u_{m}\right\}$ in $k \times \cdots \times k$ (s times) are polynomially equivalent.

3.4 Remark. In practice we shall only work with so-called tame automorphisms, i.e compositions of linear automorphisms of $k\left\{u_{1}, \ldots, u_{m}\right\}$ and 
automorphisms of the form

$$
\begin{aligned}
& u_{1} \rightarrow u_{1}, \\
& \vdots \\
& u_{i} \rightarrow a u_{i}+p\left(u_{1}, \ldots, u_{i-1}, u_{i+1}, \ldots, u_{m}\right), \\
& \vdots \\
& u_{m} \rightarrow u_{m},
\end{aligned}
$$

where $a \neq 0$ and $p$ is a polynomial in $m-1$ variables. For $m \geq 3$ it is not known whether or not every element of $\operatorname{Aut}\left(k\left\{u_{1}, \ldots, u_{m}\right\}\right)$ is tame; see [3, §4]. The difference between $\operatorname{Aut}\left(k\left\{u_{1}, \ldots, u_{m}\right\}\right)$ and its subgroup of tame automorphisms TAut $\left(k\left\{u_{1}, \ldots, u_{m}\right\}\right)$ shall not concern us here. In fact, one could replace $\operatorname{Aut}\left(k\left\{u_{1}, \ldots, u_{m}\right\}\right)$ by $\operatorname{TAut}\left(k\left\{u_{1}, \ldots, u_{m}\right\}\right)$ everywhere in this paper; all of our results would remain true and all of our arguments would go through unchanged. The same applies to $\operatorname{Aut}\left(k^{m}\right)$ versus $\operatorname{TAut}\left(k^{m}\right)$ in the statement of Theorem 3.1.

\section{Proofs of Theorems $1.2-1.4$}

As we observed in the previous section, the automorphisms of $k^{m}=Q_{m, 1}$ constructed in the proof of Theorem 3.1 are all induced by automorphisms of $k\left\{u_{1}, \ldots, u_{m}\right\}$. We shall now show that elements of $\operatorname{Aut}\left(Q_{m, n}\right)$ can be constructed from automorphisms of $k\left\{u_{1}, \ldots, u_{m}\right\}$ for any $n$.

For $\sigma \in \operatorname{End}\left(k\left\{u_{1}, \ldots, u_{m}\right\}\right)$ we define $\sigma^{*}$ by

$$
\begin{gathered}
\sigma^{*}: M_{n}(k)^{m} \rightarrow M_{n}(k)^{m}, \\
\left(X_{1}, \ldots, X_{m}\right) \rightarrow\left(\sigma\left(u_{1}\right)(X), \ldots, \sigma\left(u_{m}\right)(X)\right) .
\end{gathered}
$$

4.1 Proposition. Every $\sigma \in \operatorname{End}\left(k\left\{u_{1}, \ldots, u_{m}\right\}\right)$ induces an algebraic morphism $\sigma_{*}: Q_{m, n} \rightarrow Q_{m, n}$ such that the following diagram is commutative.

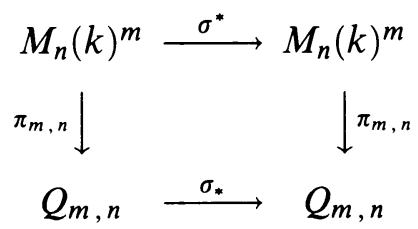

Moreover, if $\sigma$ is an automorphism of $k\left\{u_{1}, \ldots, u_{m}\right\}$ then $\sigma_{*}$ is a representation type preserving automorphism of $Q_{m, n}$.

Proof. Since $\sigma^{*}$ is $P G L_{n}$-invariant, the existence and uniqueness of $\sigma_{*}$ follows from Lemma 2.2. By uniqueness,

$$
\left(\sigma_{1} \circ \sigma_{2}\right)_{*}=\left(\sigma_{1}\right)_{*} \circ\left(\sigma_{2}\right)_{*}
$$

and

$$
(\mathrm{id})_{*}=\mathrm{id}: Q_{m, n} \rightarrow Q_{m, n} \text {. }
$$

Thus if $\sigma$ is an automorphism of $k\left\{u_{1}, \ldots, u_{m}\right\}$ then $\sigma_{*}$ is an automorphism of $Q_{m, n}$.

It remains to show that for $\sigma \in \operatorname{Aut}\left(k\left\{u_{1}, \ldots, u_{m}\right\}\right), \sigma_{*}$ preserves the representation type, i.e. for any $x \in Q_{m, n}$

$$
\tau\left(\sigma_{*} x\right)=\tau(x) \text {. }
$$


For any $\sigma \in \operatorname{End}\left(k\left\{u_{1}, \ldots, u_{m}\right\}\right)$ the subalgebra of $M_{n}(k)^{m}$ generated by $X_{1}, \ldots, X_{m}$ contains the subalgebra generated by $Y_{1}, \ldots, Y_{m}$ where

$$
\left(X_{1}, \ldots, X_{m}\right)=\sigma^{*}\left(Y_{1}, \ldots, Y_{m}\right) \text {. }
$$

Hence, for any $x \in C$ we have $\tau\left(\sigma_{*}(x)\right) \leq \tau(x)$; see Lemma 2.6. When $\sigma$ is an automorphism, this yields (8). Q.E.D.

As a direct corollary of our definition, $\sigma_{*}$ has the following property.

4.2 Lemma. Let $\sigma$ be an automorphism of $k\left\{u_{1}, \ldots, u_{m}\right\}$, let $x$ and $y$ be points of $Q_{m, n}(\tau)$ let $\varphi(x)$ and $\varphi(y)$ be their reduced semisimple representations; see (5). Then $\sigma_{*}(x)=y$ if and only if $\varphi(x) \circ \sigma \approx \varphi(y)$.

Proposition 4.1 says that $\operatorname{Aut}\left(k\left\{u_{1}, \ldots, u_{m}\right\}\right) \rightarrow \operatorname{Aut}\left(Q_{m, n}\right)$ is a morphism of groups. We shall prove Theorems $1.2-1.5$ by demonstrating that the image of this map is already big enough to have the required transitivity properties. In order to do this we will need the following generalizations of Proposition 3.3.

Recall that a representation $\rho: k\left\{u_{1}, \ldots, u_{m}\right\} \rightarrow M$ is saturated if $\rho\left(u_{1}\right)$, $\ldots, \rho\left(u_{m-1}\right)$ generate $M$ as a $k$-algebra.

4.3 Theorem. Let $M$ be a finite-dimensional semisimple $k$-algebra and let $m \geq 3$. Then any two saturated representations of $k\left\{u_{1}, \ldots, u_{m}\right\}$ in $M$ are polynomially equivalent.

4.4 Theorem. Let $M$ be a semisimple algebra,

$$
M=M_{d_{1}}(k) \times \cdots \times M_{d_{r}}(k)
$$

and let $m \geq \max \left\{d_{1}, \ldots, d_{r}\right\}+1$. Then any two irreducible representations of $k\left\{u_{1}, \ldots, u_{m}\right\}$ in $M$ are polynomially equivalent.

We shall defer the proofs of these theorems to $\S \S 6,7$ and 8 . In the remainder of this section we will demonstrate how Theorems $1.2,1.3$ and 1.4 can be derived from Theorems 4.3 and 4.4. In order to do this in the case of Theorem 1.5 we need a more elaborate argument which will be presented in the next section.

Proof of Theorem 1.2. Let $\tau=\left(e_{1}, d_{1} ; \ldots ; e_{r}, d_{r}\right)$ be a representation type and let $M$ be as in (9). Choose $x$ and $y$ in $Q_{m, n}(\tau)$. As we observed in 2.5, the reduced semisimple representations $\varphi(x)$ and $\varphi(y): k\left\{u_{1}, \ldots, u_{m}\right\} \rightarrow M$ are irreducible. Hence, by Theorem 4.4 they are polynomially equivalent, i.e. $\varphi(y)=\varphi(x) \circ \sigma$. By Lemma 4.2 this implies $\sigma_{*} x=y$, as desired. Q.E.D.

Proof of Theorem 1.4. Let $U_{0}$ be the set of points $x$ in $Q_{m, n}(\tau)$ such that $\varphi(x)$ is saturated. By Lemma $2.11 U_{0}$ is a nonempty Zariski-open subset of $Q_{m, n}(\tau)$ (here we use the assumption that $m \geq 3$ !). Let $x, y \in U_{0}$. By Theorem $4.3 \varphi(x)$ and $\varphi(y)$ are polynomially equivalent, i.e. $\varphi(y)=\varphi(x) \circ \sigma$ for some $\sigma \in \operatorname{Aut}\left(k\left\{u_{1}, \ldots, u_{m}\right\}\right)$. By Lemma $4.2 \sigma_{*}(x)=y$. Thus $\operatorname{Aut}\left(Q_{m, n}\right)$ acts transitively on $U_{0}$. Unfortunately, $U_{0}$ is not $\operatorname{Aut}\left(Q_{m, n}\right)$-invariant. This problem, however, can be corrected by defining $U_{m, n}(\tau)$ as the union of $g U_{0}$ over all $g$ in $\operatorname{Aut}\left(Q_{m, n}\right)$. Q.E.D.

Proof of Theorem 1.3. We use a diagonal argument. Let $x_{1}, \ldots, x_{s}$ and $y_{1}$, $\ldots, y_{s}$ be two $m$-tuples of distinct elements of $Q_{m, n}(1, n)$. Denote their associated irreducible representations by $\varphi\left(x_{1}\right), \ldots, \varphi\left(x_{s}\right)$ and $\varphi\left(y_{1}\right), \ldots, \varphi\left(y_{s}\right)$ : 
$k\left\{u_{1}, \ldots, u_{m}\right\} \rightarrow M_{n}(k)$ respectively. By our assumption $\varphi\left(x_{i}\right) \not \approx \varphi\left(x_{j}\right)$ and $\varphi\left(y_{i}\right) \not \varphi\left(y_{j}\right)$ for $i \neq j$. Then by Lemma 2.4,

$$
\rho_{x}=\varphi\left(x_{1}\right) \oplus \cdots \oplus \varphi\left(x_{s}\right) \quad \text { and } \quad \rho_{y}=\varphi\left(y_{1}\right) \oplus \cdots \oplus \varphi\left(y_{s}\right)
$$

are irreducible representations of $k\left\{u_{1}, \ldots, u_{m}\right\}$ in $M_{n}(k) \times \cdots \times M_{n}(k)$ (s times). By Theorem $4.4 \rho_{y}=\rho_{x} \circ \sigma$ for some $\sigma \in \operatorname{Aut}\left(k\left\{u_{1}, \ldots, u_{m}\right\}\right)$. Then for $i=1, \ldots, s \varphi\left(x_{i}\right)=\varphi\left(y_{i}\right) \circ \sigma$, and hence by Lemma 4.2, $\sigma_{*}\left(x_{i}\right)=$ $y_{i}$. Q.E.D.

\section{Proof OF TheOREM 1.5}

Let $U_{m, n}$ be the set of points $x \in Q_{m, n}(1, n)$ such that the associated irreducible representation $\varphi(x)$ is saturated. By Lemma 2.11 this set is Zariskiopen and nonempty when $m \geq 3$. We are going to prove that it has the property claimed in the statement of Theorem 1.5.

Let $x_{1}, \ldots, x_{s}$ and $y_{1}, \ldots, y_{s}$ be two $m$-tuples of distinct elements of $U$ and let $\rho_{x}$ and $\rho_{y}$ be as in (10). It is sufficient to prove that $\rho_{x}$ and $\rho_{y}$ are polynomially equivalent; as we saw in the proof of Theorem $1.3 \rho_{y}=\rho_{x} \circ \sigma$ implies $y_{i}=\sigma_{*}\left(x_{i}\right)$ for $i=1, \ldots, s$. The polynomial equivalence of $\rho_{x}$ and $\rho_{y}$, however, does not follow directly from Theorem 4.3. Indeed, while the representations $\varphi\left(x_{1}\right), \ldots, \varphi\left(x_{s}\right)$ are pairwise inequivalent, their restrictions to $k\left\{u_{1}, \ldots, u_{m-1}\right\}$ do not need to be. If they are not then $\rho_{x}$ will not be saturated, and Theorem 4.3 will not apply. The situation is saved by Proposition 5.1 below. This proposition says that we can choose $\alpha$ and $\beta$ in $\operatorname{Aut}\left(k\left\{u_{1}, \ldots, u_{m}\right\}\right)$ such that

(i) $\varphi\left(x_{i}\right) \circ \alpha$ and $\varphi\left(y_{i}\right) \circ \beta$ are saturated for $i=1, \ldots, s$,

(ii) the restricitons of $\varphi\left(x_{i}\right) \circ \alpha$ to $k\left\{u_{1}, \ldots, u_{m-1}\right\}$ are pairwise inequivalent,

(iii) the restrictions of $\varphi\left(y_{i}\right) \circ \beta$ to $k\left\{u_{1}, \ldots, u_{m-1}\right\}$ are pairwise inequivalent.

By Lemma $2.4 \rho_{x} \circ \alpha$ and $\rho_{y} \circ \beta$ are saturated representations of $k\left\{u_{1}, \ldots, u_{m}\right\}$ in $M_{n}(k) \times \cdots \times M_{n}(k)$ (s times). By Theorem $4.3 \rho_{x} \circ \alpha$ and $\rho_{y} \circ \beta$ are polynomially equivalent. Since polynomial equivalence is a transitive relation, this implies that $\rho_{x}$ and $\rho_{y}$ are polynomially equivalent, thus proving Theorem 1.5.

5.1 Proposition. For $i=1, \ldots, s$ let $\phi_{i}$ be a saturated representation of $k\left\{u_{1}, \ldots, u_{m}\right\}$ in $M_{d_{i}}(k)$. Assume that $\phi_{1}, \ldots, \phi_{s}$ are pairwise inequivalent. Then there exists $a \sigma \in \operatorname{Aut}\left(k\left\{u_{1}, \ldots, u_{m}\right\}\right)$ such that

(i) $\phi_{i} \circ \sigma$ is saturated for $i=1, \ldots, s$ and

(ii) the restrictions of $\phi_{i} \circ \sigma$ to $k\left\{u_{1}, \ldots, u_{m-1}\right\}$ are pairwise inequivalent.

Proof. Denote the restriction of a representation $\rho$ to $k\left\{u_{1}, \ldots, u_{m-1}\right\}$ by $r(\rho)$. Let $S\left(\phi_{1}, \ldots, \phi_{s}\right)$ be the set of pairs $(i, j)$ such that $i \neq j$ and $r\left(\phi_{i}\right) \approx r\left(\phi_{j}\right)$. Without loss of generality we can make the following minimality assumption on $\phi_{1}, \ldots, \phi_{s}$.

Minimality Assumption. For any $\sigma \in \operatorname{Aut}\left(k\left\{u_{1}, \ldots, u_{m}\right\}\right)$ such that each $\phi_{i} \circ \sigma$ is saturated $\left|S\left(\phi_{1}, \ldots, \phi_{s}\right)\right| \leq\left|S\left(\phi_{1} \circ \sigma, \ldots, \phi_{s} \circ \sigma\right)\right|$. 
Since the representations $r\left(\phi_{i}\right)$ are irreducible, $r\left(\phi_{i}\right) \approx r\left(\phi_{j}\right)$ if and only if $d_{i}=d_{j}$ and $\phi_{i}, \phi_{j}$ map to the same point in $Q_{m-1, d_{i}}$. Hence,

$$
S\left(\phi_{1} \circ \sigma, \ldots, \phi_{s} \circ \sigma\right)=S\left(\phi_{1}, \ldots, \phi_{s}\right)
$$

for $\sigma$ "close" to the identity automorphism of $k\left\{u_{1}, \ldots, u_{m}\right\}$.

5.2 Lemma. If $(a, b) \in S\left(\phi_{1}, \ldots, \phi_{s}\right)$ then for every $w \geq 1$,

$$
\operatorname{Tr} \phi_{a}\left(u_{m}^{w}\right)=\operatorname{Tr} \phi_{b}\left(u_{m}^{w}\right) .
$$

Proof. Assume the lemma fails for some $w \geq 1$. Define $\sigma$ by

$$
\begin{aligned}
& u_{1} \rightarrow u_{1}+c u_{m}^{w} \\
& u_{2} \rightarrow u_{2} \\
& \vdots \\
& u_{m} \rightarrow u_{m} .
\end{aligned}
$$

Then for a generic choice of $c \in k, r\left(\phi_{i} \circ \sigma\right) \not \approx r\left(\phi_{j} \circ \sigma\right)$ whenever $(i, j) \notin$ $S\left(\phi_{1}, \ldots, \phi_{s}\right)$ and every $\phi_{i} \circ \sigma$ will remain saturated. Moreover, when $c \neq 0$ $r\left(\phi_{a} \circ \sigma\right) \not \approx r\left(\phi_{b} \circ \sigma\right)$, since $\operatorname{Tr} \phi_{a} \circ \sigma\left(u_{1}^{w}\right) \neq \operatorname{Tr} \phi_{b} \circ \sigma\left(u_{m}^{w}\right)$. This means that

$$
S\left(\phi_{1} \circ \sigma, \ldots, \phi_{s} \circ \sigma\right) \subset S\left(\phi_{1}, \ldots, \phi_{s}\right) \backslash\{(a, b)\},
$$

contradicting our minimality assumption. Q.E.D.

5.3 Lemma. If $(a, b) \in S\left(\phi_{1}, \ldots, \phi_{s}\right)$ then $\operatorname{Tr} \phi_{a}\left(u_{m} p\right) \neq \operatorname{Tr} \phi_{b}\left(u_{m} p\right)$ for some $p \in k\left\{u_{1}, \ldots, u_{m-1}\right\}$.

Proof. By our assumption $d_{a}=d_{b}$ and there exists an invertible $d_{a} \times d_{a^{-}}$ matrix $g$ such that $\phi_{b}\left(u_{i}\right)=g^{-1} \phi_{a}\left(u_{i}\right) g$ for $i=1, \ldots, m-1$. Since $\phi_{a}$ and $\phi_{b}$ are not equivalent, $\phi_{a}\left(u_{m}\right) \neq g^{-1} \phi_{b}\left(u_{m}\right) g$. Recall that the Killing bilinear form $(x, y)=\operatorname{Tr}(x y)$ is nonsingular on $M_{d_{a}}(k)$. Therefore, there exists an $H \in M_{d_{a}}(k)$ such that $\operatorname{Tr}\left(\phi_{b}\left(u_{m}\right) H\right) \neq \operatorname{Tr}\left(g^{-1} \phi_{a}\left(u_{m}\right) g H\right)$. Since $\phi_{b}$ is saturated, we can write $H$ as $\phi_{b}(p)$ for some $p \in k\left\{u_{1}, \ldots, u_{m-1}\right\}$. By our assumption $H=\phi_{b}(p)=g^{-1} \phi_{a}(p) g$. Thus

$$
\begin{aligned}
\operatorname{Tr} \phi_{b}\left(u_{m} p\right) & =\operatorname{Tr} \phi_{b}\left(u_{m}\right) H \neq \operatorname{Tr} g^{-1} \phi_{a}\left(u_{m}\right) g H \\
& =\operatorname{Tr} g^{-1} \phi_{a}\left(u_{m}\right) g g^{-1} \phi_{a}(p) g=\operatorname{Tr} \phi_{a}\left(u_{m} p\right),
\end{aligned}
$$

as desired. Q.E.D.

We can now finish the proof of Proposition 5.1. Assume that the Proposition fails, i.e. $S\left(\phi_{1}, \ldots, \phi_{s}\right) \neq \varnothing$. Let

$$
(a, b) \in S\left(\phi_{1}, \ldots, \phi_{s}\right) .
$$

Choose $p\left(u_{1}, \ldots, u_{m-1}\right)$ as in Lemma 5.3 and let $\sigma$ be the automorphism of $k\left\{u_{1}, \ldots, u_{m}\right\}$ given by

$$
\begin{aligned}
& u_{1} \rightarrow u_{1}, \\
& \vdots \\
& u_{m-1} \rightarrow u_{m-1}, \\
& u_{m} \rightarrow u_{m}+p\left(u_{1}, \ldots, u_{m-1}\right) .
\end{aligned}
$$

Since $r\left(\phi_{i} \circ \sigma\right)=r\left(\phi_{i}\right)$ for $i=1, \ldots, s$, 
(i) $\phi_{i} \circ \sigma$ is saturated of $i=1, \ldots, s$ and

(ii) $S\left(\phi_{1} \circ \sigma, \ldots, \phi_{s} \circ \sigma\right)=S\left(\phi_{1}, \ldots, \phi_{s}\right)$.

Hence, $\phi_{1} \circ \sigma, \ldots, \phi_{s} \circ \sigma$ satisfy our minimality assumption. On the other hand,

(iii) By Lemma $5.2 \operatorname{Tr} \phi_{a}\left(u_{m}^{w}\right)=\operatorname{Tr} \phi_{b}\left(u_{m}^{w}\right)$ for any positive integer $w$. We will use this below with $w=2$.

(iv) $\operatorname{Tr} \phi_{a}(q)=\operatorname{Tr} \phi_{b}(q)$ for any $q \in k\left\{u_{1}, \ldots, u_{m-1}\right\}$ because $(a, b) \in$ $S\left(\phi_{1}, \ldots, \phi_{s}\right)$. We will use this below with $q=p^{2}$.

(iv) By Lemma 5.2 applied to $\phi_{1} \circ \sigma, \ldots, \phi_{s} \circ \sigma, \operatorname{Tr} \phi_{a} \circ \sigma\left(u_{m}^{w}\right)=\operatorname{Tr} \phi_{b} \circ \sigma\left(u_{m}^{w}\right)$ for any positive integer $w$. We will use this below with $w=2$.

Putting (iii), (iv) and (v) together, we obtain

$$
\begin{aligned}
2 \operatorname{Tr} \phi_{a}\left(u_{m} p\right) & =\operatorname{Tr} \phi_{a} \circ \sigma\left(u_{m}^{2}\right)-\operatorname{Tr} \phi_{a}\left(p^{2}\right)-\operatorname{Tr} \phi_{a}\left(u_{m}^{2}\right) \\
& =\operatorname{Tr} \phi_{b} \circ \sigma\left(u_{m}^{2}\right)-\operatorname{Tr} \phi_{b}\left(p^{2}\right)-\operatorname{Tr} \phi_{b}\left(u_{m}^{2}\right)=2 \operatorname{Tr} \phi_{b}\left(u_{m} p\right),
\end{aligned}
$$

contradicting our choice of $p$. Q.E.D.

\section{PolynOMIAL EQUiVALENCE OF SATURATED REPRESENTATIONS}

We now turn to the proof of Theorem 4.3. Let $\operatorname{Gr}(d)$ be the disjoint union of all grassmannians $\operatorname{Gr}(l, d)$ for $l=1, \ldots, d-1$. For $d \times d$-matrices $X_{1}, \ldots, X_{s}$ we define $V\left(X_{1}, \ldots, X_{s}\right)$ to be the subvariety of $\operatorname{Gr}(d)$ consisting of all subspaces of $k^{d}$ simultaneously invariant under the action of $X_{1}, \ldots, X_{s}$. Recall that by Burnside's theorem $X_{1}, \ldots, X_{s}$ generate $M_{d}(k)$ if and only if $V\left(X_{1}, \ldots, X_{s}\right)=\varnothing$.

6.1 Proposition. Suppose $X_{1}, \ldots, X_{m}$ generate $M_{d}(k)$. Let $W$ be a subvariety of $\operatorname{Gr}(d)$ of dimension $e$. Then for $s$ generic linear combinations $Y_{1}, \ldots, Y_{s}$ of $X_{1}, \ldots, X_{m}$,

$$
\operatorname{dim} V\left(Y_{1}, \ldots, Y_{s}\right) \cap W \leq e-s .
$$

Here as usual, $\operatorname{dim} \varnothing=-\infty$. In particular, for $e+1$ generic linear combinations $Y_{1}, \ldots, Y_{e+1}$ of $X_{1}, \ldots, X_{m}$ we have $V\left(Y_{1}, \ldots, Y_{e+1}\right) \cap W=\varnothing$.

Proof. It is enough to show that if $Y=a_{1} X_{1}+\cdots+a_{m} X_{m}$ then

$$
\operatorname{dim} V(Y) \cap W \leq \operatorname{dim} W-1
$$

for a generic choice of $a_{1}, \ldots, a_{m}$.

Choose a finite collection of points $w_{1}, \ldots, w_{N} \in W$ such that there is at least one in each irreducible component. Since $X_{1}, \ldots, X_{m}$ have no common invariant subspaces in $k^{d}$, for each $w_{i}$ there is an $X_{j}$ which does not preserve it. Hence, a generic linear combination of $X_{1}, \ldots, X_{m}$ does not preserve any $w_{i}$. In other words, for a generic choice of $a_{1}, \ldots, a_{m}$,

$$
w_{1}, \ldots, w_{N} \notin V(Y)
$$

which immediately implies the inequality (11). Q.E.D.

Let $M=M_{d_{1}}(k) \times \cdots \times M_{d_{r}}(k)$. In the sequel we will think of elements of $M$ as block-diagonal matrices of size $d_{1}+\cdots+d_{r}$. 
6.2 Lemma. (a) Suppose $X_{1}, \ldots, X_{m}$ generate $M_{d}(k)$. Assume that $X_{1}$ has distinct eigenvalues. Then for a generic choice of $a_{2}, \ldots, a_{m}$, the two matrices $X_{1}$ and $Y=a_{1} X_{1}+\cdots+a_{m} X_{m}$ generate $M_{d}(k)$.

(b) Suppose $X_{1}, \ldots, X_{m}$ generate $M=M_{d_{1}}(k) \times \cdots \times M_{d_{r}}(k)$. Assume that $X_{1}$ has distinct eigenvalues. Then for a generic choice of $a_{2}, \ldots, a_{m}$, the two elements $X_{1}$ and $Y=a_{1} X_{1}+\cdots+a_{m} X_{m}$ generate $M$.

Proof. A matrix with distinct eigenvalues has only finitely many invariant subspaces. That is, $W=V\left(X_{1}\right)$ is zero-dimensional. Proposition 6.1 says that for a generic choice of the $a_{i}-s, V\left(X_{1}\right) \cap V(Y)=\varnothing$. This proves part (a). Part (b) follows from (a) and Lemma 2.4. Q.E.D.

We are now ready to finish the proof of Theorem 4.3. The remaining part of the argument is just a more elaborate version of our proof of Theorem 3.1. Let $M$ be as in (9).

Choose $r$ diagonal matrices $A_{1}, \ldots, A_{r}$ such that $A_{j} \in M_{d_{j}}$, each $A_{j}$ has distinct eigenvalues, and $A_{j}$ and $A_{l}$ have no common eigenvalues when $j \neq l$. Let

$$
A=\left(\begin{array}{ccc}
A_{1} & & 0 \\
& \ddots & \\
0 & & A_{r}
\end{array}\right) .
$$

Now choose $B \in M$ so that $A$ and $B$ generate $M$. This can be done, for example, by following the recipe of Proposition 6.2(b).

Let $\rho=\left(X_{1}, \ldots, X_{m}\right)$ be a saturated representation of $k\left\{u_{1}, \ldots, u_{m}\right\}$ in $M$. In order to prove Theorem 4.3 it is enough to show that $\rho$ is polynomially equivalent to $(A, B, 0, \ldots, 0)$. We shall do this in five steps.

Step 1. We may assume without loss of generality that $X_{m}=A$.

Proof. Since $\rho$ is saturated, there exists a $p \in k\left\{u_{1}, \ldots, u_{m-1}\right\}$ such that $\rho(p)=A-X_{m}$. Applying the automorphism

$$
\begin{aligned}
& u_{1} \rightarrow u_{1}, \\
& \vdots \\
& u_{m-1} \rightarrow u_{m-1}, \\
& u_{m} \rightarrow u_{m}+p\left(u_{1}, \ldots, u_{m-1}\right)
\end{aligned}
$$

we see that $\rho$ is polynomially equivalent to $\left(X_{1}, \ldots, X_{m-1}, A\right)$. Q.E.D.

Step 2. We may assume without loss of generality that $X_{m}=A$ and $X_{m-1}$, $X_{m}$ generate $M_{n}(k)$.

Proof. By the result of Step 1 we may assume that $X_{m}=A$. Let $Y=a_{1} X_{1}+$ $\cdots+a_{m} X_{m}$. When $a_{m-1} \neq 0, \rho=\left(X_{1}, \ldots, X_{m}\right)$ is polynomially equivalent to $\left(X_{1}, \ldots, X_{m-2}, Y, X_{m}\right)$. By Lemma $6.2(\mathrm{~b}) Y$ and $X_{m}=A$ generate $M$ for a generic choice of the coefficients $a_{i} \in k$. Q.E.D.

Step 3. We may assume without loss of generality that $X_{m}=A$ and $X_{1}=B$.

Proof. We begin with $\left(X_{1}, \ldots, X_{m}\right)$ as in Step 2. Choose a polynomial $q$ in two variables so that $q\left(X_{m-1}, A\right)=B-X_{1}$. After replacing $X_{1}$ by $X_{1}+$ $q\left(X_{m-1}, X_{m}\right)$ (here we use the assumption that $m \geq 3$ !) we will obtain an 
$m$-tuple polynomially equivalent to the original one, with $X_{1}=B$ and $X_{m}=$ A. Q.E.D.

Step 4. We may assume without loss of generality that $X_{1}=A$ and $X_{2}=B$.

Proof. Permuting $X_{1}, \ldots, X_{m}$ produces a polynomially equivalent $m$-tuple. Q.E.D.

Step 5. $\left(X_{1}, \ldots, X_{m}\right)$ is polynomially equivalent to $(A, B, 0, \ldots, 0)$.

Proof. Since $A$ and $B$ generate $M$, for each $i=3, \ldots, m$, there exists a $p_{i} \in k\left\{u_{1}, u_{2}\right\}$ such that $p_{i}(A, B)=X_{i}$. Applying the automorphism

$$
\begin{aligned}
& u_{1} \rightarrow u_{1}, \\
& u_{2} \rightarrow u_{2}, \\
& u_{3} \rightarrow u_{3}-p_{3}\left(u_{1}, u_{2}\right), \\
& \vdots \\
& u_{m} \rightarrow u_{m}-p_{m}\left(u_{1}, u_{2}\right)
\end{aligned}
$$

we arrive at the $m$-tuple $(A, B, 0, \ldots, 0)$. Q.E.D.

\section{IRREDUCIBLE SUBSPACES}

The remainder of this paper will be devoted to proving Theorem 4.4. When $m=2$ the theorem reduces to Proposition 3.3. Therefore, from now on we shall assume that $m$ is at least 3.

We begin by observing that for $m \geq \operatorname{dim}_{k}(M)$ Theorem 4.4 is an easy consequence of Theorem 4.3. Indeed, it is enough to show that an arbitrary irreducible representation $\rho=\left(X_{1}, \ldots, X_{m}\right): k\left\{u_{1}, \ldots, u_{m}\right\} \rightarrow M$ is polynomially equivalent to a saturated one. Since $X_{1}, \ldots, X_{m}$ are linearly dependent, we may assume (after permuting them if necessary) that $X_{m}$ is a linear combination of $X_{1}, \ldots, X_{m-1}$. Then $X_{1}, \ldots, X_{m-1}$ generate $M$, as desired.

If $M=M_{n}(k)$, we can do a little better by applying Proposition 6.1 with $W=\operatorname{Gr}(n)$. The dimension of $\operatorname{Gr}(n)$ is $\left[n^{2} / 4\right]$ where $\left[n^{2} / 4\right]$ denotes the integral part of $n^{2} / 4$. For $m>\left[n^{2} / 4\right]+1$, Proposition 6.1 says that after a linear change of variables we may assume that $V\left(X_{1}, \ldots, X_{m-1}\right)=\varnothing$. In other words, $\left(X_{1}, \ldots, X_{m}\right)$ is saturated, and we can apply Theorem 4.3. This proves Theorem 4.4 in the case $M=M_{n}(k)$ and $m \geq\left[n^{2} / 4\right]+2$. Our proof of Theorem 4.4 in the general case is based on a more careful argument along these lines. The main improvement will be a better choice of $W$ in the application of Proposition 6.1. Instead of trying to eliminate all invariant subspaces of $X_{1}, \ldots, X_{m-1}$ we shall only focus on the irreducible ones; see the proof of Proposition 8.3. In the remainder of this section we shall discuss some of the properties of irreducible subspaces for a given representation $\phi$.

Let $\phi: k\left\{u_{1}, \ldots, u_{l}\right\} \rightarrow M_{n}(k)$ be a representation and let

$$
(0)=V_{0} \subset V_{1} \subset \cdots \subset V_{q}=k^{n}
$$

be a composition series of $\phi$ as in (2). Denote the induced irreducible representations of $k\left\{u_{1}, \ldots, u_{l}\right\} \rightarrow \operatorname{End}\left(V_{i} / V_{i-1}\right)$ by $\phi_{i}$.

We say that an invariant subspace $U \subset k^{n}$ is irreducible for $\phi$ if the induced representation $k\left\{u_{1}, \ldots, u_{l}\right\} \rightarrow \operatorname{End}(U)$ is irreducible, i.e. $U$ is irreducible as a $k\left\{u_{1}, \ldots, u_{l}\right\}$-module with the module structure given by $\phi$. 
Let $\alpha: k\left\{u_{1}, \ldots, u_{l}\right\} \rightarrow M_{d}(k)$ be an irreducible representation. Denote the $d$-dimensional vector space on which $M_{d}(k)$ operates by $U_{\alpha}$. We view $U_{\alpha}$ as a $k\left\{u_{1}, \ldots, u_{l}\right\}$-module via $\alpha$. Let $H_{\alpha}=\operatorname{Hom}_{k\{u\}}\left(U_{\alpha}, k^{n}\right)$ be the set of all $k\left\{u_{1}, \ldots, u_{1}\right\}$-homomorphisms from $U_{\alpha}$ to $k^{n}$ with the $k\left\{u_{1}, \ldots, u_{l}\right\}$ module structure on $k^{n}$ given by $\phi$. We will think of $H_{\alpha}$ as a $k$-vector space. In this situation Schur's lemma can be restated as follows.

7.1 Lemma. (a) Let $0 \neq f \in H_{\alpha}$. Then the image of $f$ is an irreducible $d$-dimensional subspace of $k^{n}$.

(b) Let $f, g: U_{\alpha} \rightarrow W$ be two homomorphisms of $k\left\{u_{1}, \ldots, u_{l}\right\}$-modules. Assume that $f\left(U_{\alpha}\right)=g\left(U_{\alpha}\right)$. Then $f=c g$ for some $c \in k$.

Proof. (a) Since $U_{\alpha}$ is irreducible as a $k\left\{u_{1}, \ldots, u_{l}\right\}$-module, so is $f\left(U_{\alpha}\right)$. Since $\operatorname{ker}(f)$ is a $k\left\{u_{1}, \ldots, u_{l}\right\}$-submodule of $U_{\alpha}$, it is either $(0)$ or all of $U_{\alpha}$. The case $\operatorname{ker}(f)=U_{\alpha}$ is ruled out by the assumption that $f \neq 0$. This proves part (a). To prove (b) observe that if $f\left(U_{\alpha}\right)=g\left(U_{\alpha}\right)$ then

$$
f^{-1} \circ g: U_{\alpha} \rightarrow U_{\alpha}
$$

is an endomorphism of $U_{\alpha}$ as a $k\left\{u_{1}, \ldots, u_{l}\right\}$-module. By Schur's lemma any such endomorphism is a scalar multiplication. Q.E.D.

Let $U$ be an irreducible subspace of $k^{n}$. Then $U \cap V_{i}$ is either (0) or all of $U$. The smallest value of $i$ such that $U \subset V_{i}$ will be called the level of $U$. We will say that $f \in H_{\alpha}$ is of level $i$ if $f\left(U_{\alpha}\right)$ is of level $i$.

7.2 Lemma. Let $U$ be an irreducible subspace of $k^{n}$ of level $i$. Then

(a) $U$ is isomorphic to $V_{i} / V_{i-1}$ as a $k\left\{u_{1}, \ldots, u_{l}\right\}$-module.

(b) The representation $k\left\{u_{1}, \ldots, u_{l}\right\} \rightarrow \operatorname{End}(U)$ induced by $\phi$ is equivalent to $\phi_{i}$.

(c) There exists an $f \in H_{\phi_{i}}$ such that $f\left(U_{\phi_{i}}\right)=U$.

Proof. Since $U$ is of level $i, U \subset V_{i}$ and the projection map $U \rightarrow V_{i} / V_{i-1}$ is an injective homomorphism of irreducible $k\left\{u_{1}, \ldots, u_{l}\right\}$-modules. Hence, it must be an isomorphism. Parts (b) and (c) are immediate consequences of (a). Q.E.D.

7.3 Lemma. Let $f_{1}, \ldots, f_{e}$ be nonzero elements of $H_{\alpha}$ of levels $1 \leq i_{1}<$ $\cdots<i_{e} \leq q$. Then

(a) $f_{1}, \ldots, f_{e}$ are linearly independent over $k$.

(b) Assume that there are no elements of $H_{\alpha}$ of any other level. Then $f_{1}, \ldots$, $f_{e}$ form a basis of $H_{\alpha}$.

Proof. (a) Suppose $a_{1} f_{1}+\cdots+a_{e} f_{e}=0$. By Lemma $7.1 f_{e}: U_{\alpha} \rightarrow k^{n}$ is injective and $f_{e}\left(U_{\alpha}\right)$ is irreducible. Hence, $f_{e}\left(U_{\alpha}\right) \cap V_{i_{e}-1}=(0)$ and for any $0 \neq u \in U_{\alpha}$ we have $f_{e}(u) \notin V_{i_{e}}$ while $f_{1}(u), \ldots, f_{e-1}(u) \in V_{e_{i}-1}$. This is only possible if $a_{e}=0$. Part (a) now follows by induction on $e$.

(b) Let $f$ be of level $i_{j}$. Composing $f$ (resp. $f_{j}$ ) with the projection map $V_{i} \rightarrow V_{i} / V_{i-1}$ we obtain $\tilde{f}$ (resp. $\left.\tilde{f}_{j}\right): U_{\alpha} \rightarrow V_{i_{j}} / V_{i_{j}-1}$. Since both of these maps are surjective, Lemma 7.1(b) says that $\tilde{f}=c \tilde{f}_{j}$ for some $c \in k$. This means that $f-c f_{i_{j}}$ is of a lower level than $i_{j}$. Part (b) now follows by induction on $j$. Q.E.D.

Let $\alpha: k\left\{u_{1}, \ldots, u_{l}\right\} \rightarrow M_{d}(k)$ be as above. We define $W(\phi, \alpha) \subset \operatorname{Gr}(d, n)$ 
to be the set of all irreducible subspaces of $k^{n}$ such that the representation $k\left\{u_{1}, \ldots, u_{l}\right\} \rightarrow \operatorname{End}(U)$ induced by $\phi$ is isomorphic to $\alpha$.

7.4 Lemma. Let $n_{\alpha}$ be the number of $i=1, \ldots, q$ such that $\phi_{i}$ is equivalent to $\alpha$. Then $W(\phi, \alpha)$ is a projective subvariety of $\operatorname{Gr}(d, n)$ of dimension at most $n_{\alpha}-1$.

Proof. By the definition of the algebraic structure on $\operatorname{Gr}(d, n)$ the map

$$
\begin{gathered}
\operatorname{Hom}_{k}\left(U_{\alpha}, k^{n}\right) \rightarrow \operatorname{Gr}(d, n) \\
f \rightarrow f\left(U_{\alpha}\right)
\end{gathered}
$$

is rational. Denote the restriction of this map to $H_{\alpha}$ by $F$. By Lemma 7.1(a) the image of $(0) \neq f \in H_{\alpha}$ has dimension $d$, i.e. $F$ is regular on $H_{\alpha} \backslash(0)$. By Lemma 7.2(c) the image of $F$ is precisely $W(\phi, \alpha)$. By Lemma 7.1(b) $F(f)=F(g)$ if and only if $f=c g$. Hence, $F$ induces an injective regular map

$$
\widetilde{F}: \mathbf{P}\left(H_{\alpha}\right) \rightarrow \operatorname{Gr}(d, n)
$$

whose image $W(\phi, \alpha)$ is a projective subvariety of $\operatorname{Gr}(d, n)$ is a projective subvariety of $\operatorname{Gr}(d, n)$. By Lemma 7.3(b), $\operatorname{dim}_{k} H_{\alpha} \leq n_{\alpha}$. Hence, $\operatorname{dim} W(\phi, \alpha) \leq n_{\alpha}-1$. Q.E.D.

7.5 Lemma. Let $\psi: k\left\{u_{1}, \ldots, u_{s}\right\} \rightarrow M_{n}(k)$ be a representation such that

$$
\phi\left(k\left\{u_{1}, \ldots, u_{l}\right\}\right) \subset \psi\left(k\left\{u_{1}, \ldots, u_{s}\right\}\right) \text { and } \tau(\phi)=\tau(\psi) .
$$

If a subspace $U \subset k^{n}$ is invariant and irreducible for $\psi$ then it is invariant and irreducible for $\phi$.

Proof. $U$ is invariant for $\phi$ because $\phi\left(k\left\{u_{1}, \ldots, u_{l}\right\}\right) \subset \psi\left(k\left\{u_{1}, \ldots, u_{s}\right\}\right)$. By our assumption on the representation types of $\phi$ and $\psi$, if (12) is a composition series for $\psi$ then it is also a composition series for $\phi$. Suppose $U$ is of level $i$ as an irreducible representation for $\psi$. Then the projection map $U \rightarrow V_{i} / V_{i-1}$ is an isomorphism of $k\left\{u_{1}, \ldots, u_{s}\right\}$-modules. Consider them as $k\left\{u_{1}, \ldots, u_{l}\right\}$-modules via $\phi$. Since the right-hand side is irreducible, the left-hand side must be irreducible as well. Q.E.D.

\section{Polynomial EQUIVALENCE OF IRREDUCIBLE REPRESENTATIONS}

In this section we complete the proof of Theorem 4.4.

We shall think of elements of $M=M_{d_{1}} \times \cdots \times M_{d_{r}}$ as square block-diagonal matrices of size $d=d_{1}+\cdots+d_{r}$, as we did in $\S 6$. It will thus make sense to talk about the representation type of a representation

$$
\rho=\left(X_{1}, \ldots, X_{s}\right): k\left\{u_{1}, \ldots, u_{s}\right\} \rightarrow M
$$

see 2.5. As usual, it will be denoted by $\tau(\rho)$ or $\tau\left(X_{1}, \ldots, X_{s}\right)$.

If one attempts to reduce Theorem 4.4 to Theorem 4.3 by induction on the representation type of $r(\rho)$, one naturally arrives at the following definition. if

An irreducible representation $\rho: k\left\{u_{1}, \ldots, u_{m}\right\} \rightarrow M$ will be called optimal

$$
\tau\left(X_{1}, X_{2}\right)=\max \left\{\tau\left(\rho \circ \sigma\left(u_{1}\right), \ldots, \rho \circ \sigma\left(u_{m-1}\right)\right): \sigma \in \operatorname{Aut}\left(k\left\{u_{1}, \ldots, u_{m}\right\}\right)\right\} .
$$


8.1 Proposition. Let $m \geq 3$. Then every irreducible representation of $k\left\{u_{1}, \ldots, u_{m}\right\}$ in $M$ is polynomially equivalent to an optimal representation. Proof. Let $\rho=\left(X_{1}, \ldots, X_{m}\right)$ be an irreducible representation of $k\left\{u_{1}, \ldots, u_{m}\right\}$ in $M$. We may assume without loss of generality that $\mu=\tau\left(X_{1}, \ldots, X_{m-1}\right)$ is maximal among all representation types $\tau\left(Y_{1}, \ldots, Y_{m-1}\right)$ where $\left(Y_{1}, \ldots, Y_{m}\right)$ is polynomially equivalent to $\left(X_{1}, \ldots, X_{m}\right)$.

\subsection{Lemma. Let}

$$
l=a_{1} u_{1}+\cdots+a_{m-1} u_{m-1} .
$$

There exists a $p \in k\left\{u_{1}, \ldots, u_{m-1}\right\}$ such that $\tau(\rho(p), \rho(l))=\mu$ for a generic choice of the coefficients $a_{1}, \ldots, a_{m-1} \in k$.

Proof. This lemma is a direct consequence of Lemma 6.2(b). Indeed, let

$$
\varphi: k\left\{u_{1}, \ldots, u_{m}\right\} \rightarrow N
$$

be the reduced semisimple representation associated to $r(\rho)=\left(X_{1}, \ldots, X_{m-1}\right)$. Choose an element of $N$ with distinct eigenvalues (as a block-diagonal matrix). Since $\varphi$ is irreducible, we can write this element as $\rho(p)$ for some $p \in k\left\{u_{1}, \ldots, u_{m-1}\right\}$. The assertion of the lemma follows from the fact that for a generic choice of $a_{1}, \ldots, a_{m-1} \in k, \rho(p)$ and $\rho(l)$ generate $N$; see Lemma 6.2(b). Q.E.D.

Choose $p \in k\left\{u_{1}, \ldots, u_{m-1}\right\}$ as in Lemma 8.2. We define $\sigma: k\left\{u_{1}, \ldots, u_{m}\right\}$ $\rightarrow k\left\{u_{1}, \ldots, u_{m}\right\}$ by

$$
\begin{aligned}
& u_{1} \rightarrow a_{1} u_{1}+\cdots+a_{m-1} u_{m-1}, \\
& u_{2} \rightarrow a_{m} u_{m}+p\left(u_{1}, \ldots, u_{m-1}\right) \\
& u_{3} \rightarrow u_{3} \\
& \vdots \\
& u_{m-1} \rightarrow u_{m-1} \\
& u_{m} \rightarrow u_{2}
\end{aligned}
$$

We claim that for a generic choice of the coefficients $a_{1}, \ldots, a_{m} \in k$

$$
\tau\left(\rho \circ \sigma\left(u_{1}\right), \rho \circ \sigma\left(u_{2}\right)\right) \geq \mu .
$$

Indeed, by Corollary 2.8 it is enough to show that (13) holds for one choice of $a_{1}, \ldots, a_{m}$. When $a_{m}=0$ this follows from Lemma 8.2.

Hence, we can choose the coefficients $a_{1}, \ldots, a_{i}$ so that (13) holds and $a_{1}, a_{m} \neq 0$. The latter condition insures that $\sigma$ is invertible. Since

$$
\mu \leq \tau\left(\rho \circ \sigma\left(u_{1}\right), \rho \circ \sigma\left(u_{2}\right)\right) \leq \tau\left(\rho \circ \sigma\left(u_{1}\right), \ldots, \rho \circ \sigma\left(u_{m-1}\right)\right) \leq \mu,
$$

this completes the proof of Proposition 8.1. Q.E.D.

8.3 Proposition. Let $m \geq n+1$, let

$$
\rho=\left(X_{1}, \ldots, X_{m}\right): k\left\{u_{1}, \ldots, u_{m}\right\} \rightarrow M_{n}(k)
$$

be an optimal irreducible representation, and let

$$
L: k\left\{u_{1}, \ldots, u_{m}\right\} \rightarrow k\left\{u_{1}, \ldots, u_{m}\right\}
$$


be given by

$$
\begin{aligned}
& u_{1} \rightarrow a_{11} u_{1}+\cdots+a_{1 m} u_{m}, \\
& \vdots \\
& u_{m-1} \rightarrow a_{m-11} u_{1}+\cdots+a_{m-1 m} u_{m}, \\
& u_{m} \rightarrow u_{m} .
\end{aligned}
$$

Then for a generic choice of the coefficients $a_{i j} \in k$ the representation $\rho \circ L$ is saturated.

Proof. Note that it is enough to find one such choice of $a_{i j}$. For $i=1, \ldots, m$ let $Y_{i}=a_{i 1} X_{1}+\cdots+a_{i m} X_{m}$. By Lemma 6.1,

$$
\operatorname{dim} \operatorname{Gr}(1, n) \cap V\left(Y_{1}, Y_{2}\right) \leq n-3
$$

for a generic choice of $a_{11}, \ldots, a_{1 m}, a_{21}, \ldots, a_{2 m} \in k$. Note that if $m=3$ then $n \leq 2$. In this case $Y_{1}$ and $Y_{2}$ have no common invariant 1-dimensional subspaces in $k^{n}$. Hence, they generate $M_{n}(k)$ which proves the proposition. Thus from now on we may assume that $m$ is at least 4 .

Let $\mu=\tau\left(X_{1}, X_{2}\right)=\tau\left(X_{1}, \ldots, X_{m-1}\right)$. For a generic choice of $a_{11}, \ldots$, $a_{1 m}, a_{21}, \ldots, a_{2 m} \in k$ we also have $\tau\left(Y_{1}, Y_{2}\right) \geq \mu$; see Corollary 2.8. Since $\rho$ is optimal, this is the same as

$$
\tau\left(Y_{1}, Y_{2}\right)=\mu \text {. }
$$

We fix $a_{11}, \ldots, a_{1 m}, a_{21}, \ldots, a_{2 m} \in k$ so that (15) and (16) hold.

Let $\phi=\left(Y_{1}, Y_{2}\right): k\left\{u_{1}, u_{2}\right\} \rightarrow M_{d}(k)$ and let

$$
(0)=V_{0} \subset V_{1} \subset \cdots \subset V_{q}=V
$$

be a composition series for $\phi$. Denote the irreducible representation $k\left\{u_{1}, u_{2}\right\}$ $\rightarrow \operatorname{End}\left(V_{i} / V_{i-1}\right)$ by $\phi_{i}$, as we did in $\S 7$.

Recall that in the previous section we defined $W\left(\phi, \phi_{i}\right)$ as the set of all $\phi$ irreducible $U \subset k^{n}$ such that the induced representation $k\left\{u_{1}, u_{2}\right\} \rightarrow \operatorname{End}(U)$ is equivalent to $\phi_{i}$. We define $W \subset \operatorname{Gr}(n)$ to be the union of all $W\left(\phi, \phi_{i}\right)$ with $\operatorname{dim} V_{i} / V_{i-1} \geq 2$. By Lemma $7.4 \operatorname{dim} W\left(\phi, \phi_{i}\right) \leq n_{\phi_{i}}-1$ where $n_{\phi_{i}}$ is the number of $j=1, \ldots, q$ such that $\phi_{j}$ is equivalent to $\phi_{i}$. If the $\operatorname{dim} V_{i} / V_{i-1}$ is at least 2 then $n_{\phi_{i}} \leq[n / 2]$. Hence,

$$
\operatorname{dim} W\left(\phi, \phi_{i}\right) \leq n_{\phi_{i}}-1 \leq\left[\frac{n}{2}\right]-1 \leq\left[\frac{m-1}{2}\right]-1 \leq m-4 .
$$

Therefore, $\operatorname{dim} W \leq m-4$. Let $W_{1}=\operatorname{Gr}(1, n) \cap V\left(Y_{1}, Y_{2}\right)$. By (15) $\operatorname{dim} W_{1} \leq n-3 \leq m-4$.

By Proposition 6.1,

$$
W \cap V\left(Y_{3}, \ldots, Y_{m-1}\right)=\varnothing \quad \text { and } W_{1} \cap V\left(Y_{3}, \ldots, Y_{m-1}\right)=\varnothing
$$

for a generic choice of $a_{i j}$ with $i=3, \ldots, m-1$ and $j=1, \ldots, m$. In order to prove Proposition 8.3 it is enough to show that for any such choice of $a_{i j} Y_{1}, \ldots, Y_{m-1}$ generate the matrix algebra $M_{n}(k)$.

Indeed, assume the contrary. Then $\left(Y_{1}, \ldots, Y_{m-1}\right)$ will have an irreducible invariant subspace $U \subset k^{n}$. This subspace cannot be 1-dimensional, since $\operatorname{Gr}(1, n) \cap V\left(Y_{1}, \ldots, V_{m-1}\right)=\varnothing$. By our choice of $Y_{1}$ and $Y_{2}$,

$$
\tau\left(Y_{1}, Y_{2}\right)=\tau\left(Y_{1}, \ldots, Y_{m-1}\right)=\mu \text {. }
$$


Hence, by Lemma $7.5 U$ will be invariant and irreducible for $\phi=\left(Y_{1}, Y_{2}\right)$. By Lemma 7.2(b) this implies $U \in W$, contradicting $W \cap V\left(V_{3}, \ldots, V_{m-1}\right)=$ $\varnothing$.

We can now complete the proof of Theorem 4.4. Let $M=M_{d_{1}} \times \cdots \times M_{d_{r}}$ and let

$$
\rho=\rho_{1} \oplus \cdots \oplus \rho_{r}: k\left\{u_{1}, \ldots, u_{m}\right\} \rightarrow M
$$

be an irreducible representation. Here $\rho_{i}: k\left\{u_{1}, \ldots, u_{m}\right\} \rightarrow M_{d_{i}}$ for $i=$ $1, \ldots, r$. The representations $\rho_{i}$ are irreducible and pairwise inequivalent. Recall that in view of Proposition 3.3 we are assuming that $m \geq 3$. Hence, it is enough to show that $\rho$ is polynomially equivalent to a saturated representation; then Theorem 4.3 will apply.

Let $L: k\left\{u_{1}, \ldots, u_{m}\right\} \rightarrow k\left\{u_{1}, \ldots, u_{m}\right\}$ be as in (14). Then the following conditions are satisfied for a generic choice of the coefficients $a_{i j}$.

(i) $\operatorname{det}\left(a_{i j}\right) \neq 0$, i.e. $L$ is an automorphism of $k\left\{u_{1}, \ldots, u_{m}\right\}$.

(ii) The representations $\rho_{1} \circ L, \ldots, \rho_{r} \circ L$ are saturated. Since

$$
m \geq \max \left\{d_{1}, \ldots, d_{r}\right\}+1,
$$

this follows from Proposition 8.3.

(iii) The representations $\rho_{1} \circ L, \ldots, \rho_{r} \circ L$ are pairwise inequivalent.

By Proposition $5.1 \rho \circ L$ is polynomially equivalent to a saturated representation. This completes the proof of Theorem 4.4 .

\section{ACKNOWLEDGMENTS}

This work was carried out at the Mathematical Sciences Research Institute in Berkeley while the author was supported by an NSF Postdoctoral Fellowship. The author is deeply grateful to MSRI for its hospitality and to the NSF for its financial support. The author also wishes to thank R. Guralnick for helpful communications.

\section{REFERENCES}

1. M. Artin, On Azumaya algebras and finite dimensional representations of rings, J. Algebra 11 (1969), 532-563.

2. C. Bessendort and L. Le Bruyn, Stable rationality of certain $P G L_{n}$ quotients, Invent. Math. 104 (1991), 179-199.

3. P. M. Cohn, Free associative algebras, Israel J. Math. 19 (1974), 109-151.

4. E. Formanek, The center of the ring of $3 \times 3$ generic matrices, Linear and Multilinear Algebra 7 (1979), 203-212.

5. _ The center of the ring of $4 \times 4$ generic matrices, J. Algebra 62 (1980), 304-319.

6. __ Noncommutative invariant theory, Group Actions on Rings, Contemp. Math., vol. 13, Amer. Math. Soc., Providence, R.I., 1985.

7. P. I. Katsylo, Stable rationality of fields of invariants of linear representations of the groups $P G L_{6}$ and $P G L_{12}$, Mat. Zametki 48 (1990), 49-52. (Russian)

8. L. Le Bruyn, Simultaneous equivalence of square matrices, Séminaire d'Algèbre Paul Dubreil et Marie-Paul Malliavin, Lecture Notes in Math., vol. 1404, Springer-Verlag, Berlin and Heidelberg, 1989.

9. L. Le Bruyn and C. Procesi, Étale local structure of matrix invariants and concomitants, Algebraic Groups Utrecht 86, Lecture Notes in Math., vol. 1271, Springer-Verlag, Berlin, Heidelberg, and New York, 1986. 
10. D. Mumford and J. Fogarty, Geometric invariant theory, Springer-Verlag, Berlin, Heidelberg, and New York, 1982.

11. C. Procesi, Non-commutative affine rings, Atti Accad. Naz. Lincei Mem. Cl. Sci. Fis. Mat. Natur. Seq. I 86 (1967), 239-255.

12. _ The invariant theory of $n \times n$ matrices, Adv. in Math. 19 (1976), 306-381.

13. Z. Reichstein, A functorial interpretation of the ring of matrix invariants, J. A.lgebra 136 (1991), 439-462.

14. L. H. Rowen, Polynomial identities in ring theory, Academic Press, New York, 1980.

15. A. H. Schofield, Matrix invariants of composite size, J. Algebra 147 (1992), 345-349.

16. G. Schwartz, Lifting smooth homotopies of orbit spaces, Inst. Hautes Études Sci. Publ. Math. 51 (1980), 37-135.

17. K. S. Sibirskii, Algebraic invariants of a set of matrices, Sibirsk Mat. Ž. 9(1) (1968), 152-164 (Russian); English transl. in Siberian Math. J. 9 (1968), 115-124.

Department of Mathematics, University of California Berkeley, California 94720

Current address: Department of Mathematics, Oregon State University, Corvallis, Oregon 97331-4605

E-mail address: zinovy@math.orst.edu 